\title{
Cortical and Leptomeningeal Cerebrovascular Amyloid and White Matter Pathology in Alzheimer's Disease
}

\author{
Alex E Roher, ${ }^{1}$ Yu-Min Kuo, ${ }^{2}$ Chera Esh, $^{1}$ CARMen Knebel, $^{1}$ Nicole Weiss, ${ }^{1}$ \\ Walter Kalback, ${ }^{1}$ DeAn C Luehrs, ${ }^{1}$ Jennifer L Childress, ${ }^{1}$ Thomas G Beach, ${ }^{3}$ \\ ROY O WELLER, ${ }^{4}$ AND TYLER A KOKJOHN ${ }^{5}$
}

\begin{abstract}
Alzheimer's disease (AD) is characterized by neurofibrillary tangles and by the accumulation of $\beta$-amyloid (A $\beta$ ) peptides in senile plaques and in the walls of cortical and leptomeningeal arteries as cerebral amyloid angiopathy (CAA). There also is a significant increase of interstitial fluid (ISF) in cerebral white matter (WM), the pathological basis of which is largely unknown. We hypothesized that the accumulation of ISF in dilated periarterial spaces of the WM in AD correlates with the severity of CAA, with the total A $\beta$ load in the cortex and with Apo E genotype. A total of 24 AD brains and 17 nondemented age-matched control brains were examined. CAA was seen in vessels isolated from brain by using EDTA-SDS lysis stained by Thioflavin-S. Total A $\beta$ in gray matter and WM was quantified by immunoassay, ApoE genotyping by PCR, and dilatation of perivascular spaces in the WM was assessed by quantitative histology. The study showed that the frequency and severity of dilatation of perivascular spaces in the WM in AD were significantly greater than in controls $(P<0.001)$ and correlated with $A \beta$ load in the cortex, with the severity of CAA, and with ApoE $\& 4$ genotype. The results of this study suggest that dilation of perivascular spaces and failure of drainage of ISF from the WM in AD may be associated with the deposition of A $\beta$ in the perivascular fluid drainage pathways of cortical and leptomeningeal arteries. This failure of fluid drainage has implications for therapeutic strategies to treat Alzheimer's disease.
\end{abstract}

\section{INTRODUCTION}

Alzheimer's disease (AD) is characterized clinically by dementia and pathologically by the accumulation of ubiquitinated tau and other proteins within neurons and by deposition of $\beta$-amyloid $(\mathrm{A} \beta)$ in the extracellular compartments of gray matter (GM) and in blood vessel walls as cerebral amyloid angiopathy (CAA) (1-3). Tracer studies in experimental animals have shown that in CAA, $\mathrm{A} \beta$ accumulates within the perivascular channels around capillaries and arteries that have been identified as interstitial fluid (ISF) drainage pathways (4-11). Biochemical determinations have shown that it is the levels of soluble $A \beta$ in Alzheimer brains, rather than the insoluble $A \beta$ plaque load, that correlate with severity of cognitive decline $(12,13)$. These findings support the hypothesis that the failure to eliminate $A \beta$ along ISF drainage pathways is a major factor in $A \beta$ accumulation in the cortex and in the $\mathrm{AD}$ pathogenesis.

In addition to the pathological changes in cerebral gray matter $(\mathrm{GM})$ in $\mathrm{AD}$, there is significant pathology in the underlying cerebral white matter (WM). This is characterized by a relative increase in ISF detectable through imaging and by histological techniques (14). There is some evidence that vascular insufficiency may be involved in the pathogenesis of WM changes in AD (15). However, there also is the distinct possibility that deposition of $A \beta$ in periarterial ISF drainage pathways may interfere with the drainage of ISF from cerebral WM particularly as dilation of periarterial spaces (état criblé) is a feature of white matter in AD.

Cerebral cortex and underlying WM both receive their blood supply from branches of leptomeningeal arteries on the surface of the brain. Extensive studies by Duvernoy and others (16) have shown that arteries supplying the cerebral hemispheres penetrate the cortex perpendicular to the surface and exhibit various branching patterns and capillary fields within the cortex. Some arteries supply the WM branch as they pass through the cortex, but others do not. The arteries supplying the WM, therefore, are derived from the same leptomeningeal arteries that supply the overlying cortex and exhibit prominent CAA in AD.

CAA is a feature of a number of familial diseases and is present in the brains of the majority if not all patients with AD (15). The pattern of deposition of $A \beta$ in capillary and artery walls correlates with the ISF drainage pathways for interstitial fluid (17). A major risk factor for CAA is possession of the ApoE $\varepsilon 4$ genotype (18).

In the present study, we test the hypothesis that accumulation of fluid in dilated periarterial ISF pathways in the WM in AD correlates with severity of CAA, total $\mathrm{A} \beta$ load in the overlying cortex, and Apo E genotype. In order to test this hypothesis, we employed a novel strategy to analyze the topology of cerebrovascular amyloid that took advantage of the extreme insolubility and

\footnotetext{
${ }^{1}$ The Longtine Center for Molecular Biology and Genetics, Sun Health Research Institute, Sun City, AZ 85351 ; ${ }^{2}$ Department of Cell Biology and Anatomy, National Cheng Kung University, Tainan, Taiwan 701; ${ }^{3}$ The Harold Civin Laboratory of Neuropathology, Sun Health Research Institute, Sun City, AZ 85351; “Department of Pathology (Neuropathology), University of Southampton School of Medicine, Southampton General Hospital, Southampton, UK; and 5Department of Microbiology, Midwestern University, Glendale, AZ 85308.
} 
resistance to disruption of amyloid deposits. This technique involves the combined action of EDTA-SDS lysis and allows detailed analysis of entire tufts of blood vessels spanning the GM. Total A $\beta$ peptide in GM and WM was quantified by immunoassay and ApoE genotyping by PCR. The dilation of perivascular spaces in the WM was assessed by quantitative histology.

\section{MATERIALS AND METHODS}

\section{Human Subjects - Brain Tissue}

Brain tissue was obtained from the Brain Donation Program of the Sun Health Research Institute. The cases were chosen to provide a mixture of Apo E genotypes in both control nondemented (ND) and $\mathrm{AD}$ groups. Characteristics of the cases in this study are given in Table 1 and include age, Apo E genotype, density of neocortical neuritic plaques, Braak stage, and the National Institute of Aging (NIA)-Reagan Institute diagnostic status. The terms "high" and "intermediate" reflect the probability that symptoms of dementia were due to AD. The mean age of ND cases was $83.2 \mathrm{y}$, whereas that of AD cases was 82.2 y. Nondemented cases $(n=17)$ comprised 8 females and 9 males while the AD cohort $(n=24)$ contained 11 females and 13 males. Mean postmortem intervals were $2.6 \mathrm{~h}$ for ND subjects and $2.8 \mathrm{~h}$ for AD subjects (difference not significant on 2-tailed unpaired $t$-test). Leptomeninges were dissected from the brain surface in the immediate post-mortem period and stored at $-80{ }^{\circ} \mathrm{C}$. Cerebral hemispheres were cut in the coronal plane as 8 - to 10 -mm thick slices. Right hemisphere slices were frozen between sheets of dry ice and stored at $-80^{\circ} \mathrm{C}$.

\section{Tissue Processing}

Left hemisphere slices were fixed in $4 \%$ paraformaldehyde for $48 \mathrm{~h}$ and then dissected into small tissue blocks for paraffin embedding or larger blocks for microtome sectioning. Paraffin blocks were sectioned at $5 \mu \mathrm{m}$ and stained with hematoxylin and eosin (H\&E). Frozen sections $(40 \mu \mathrm{m})$ were cut after cryoprotection in $2 \%$ dimethyl sulphoxide and $20 \%$ glycerol using a sledge-type microtome, stained with H\&E for general morphology and observation of WM. The lesions of $\mathrm{AD}$ were visualized using the Campbell-Switzer and Gallyas stains. Brain sections also were stained with $1 \%$ aqueous (wt/vol) Thioflavin-S (Sigma, St. Louis, $\mathrm{MO}, \mathrm{USA}$ ) and rinsed 10 times with $70 \%$ ethanol to remove unbound fluorochrome. The sections were mounted with Apathy's media and observed in an epifluorescent microscope.

\section{Neuropathological Tissue and Patient Grading}

Rarefaction of the WM in each cerebral lobe was graded on the fraction of the centrum semiovale affected in thick $(40 \mu \mathrm{m})$ quarter-hemisphere sections stained with H\&E (mild $<25 \%$; moderate $25 \%$ to $50 \%$; severe $>50 \%$ ). Individual percentages for each lobe were converted to a numeric code $($ mild $=1$; moderate $=2$; severe $=3$ ) from which a mean score for each brain was obtained. Neuritic plaque density in cerebral cortex was graded according to criteria established by the Consortium to Establish a Registry for Alzheimer's Disease (1). Neurofibrillary tangles were staged according to the method of Braak (2). The diagnosis of AD was assigned whenever a subject was demented and had at least a "moderate" plaque density. In addition, the protocol developed by the NIA and the Reagan Institute (3) was used to assign to each demented case a probability estimate that dementia was due to AD.

\section{Transmission Electron Microscopy}

Cerebral cortex was taken fresh postmortem $(1.2 \mathrm{~h})$ from $3 \mathrm{AD}$ individuals, cut into small fragments (about $1 \mathrm{~mm}^{3}$ ), and processed for electron microscopy. The specimens were fixed in $3 \%$ buffered glutaraldehyde and placed in $4 \%$ unbuffered osmium tetroxide. The blocks were stained with $1 \%$ aqueous uranyl acetate for $30 \mathrm{~min}$, embedded in epon araldite resin, thin-sectioned, and treated with lead citrate. The specimens were examined by a Phillips 400 electron microscope at $80 \mathrm{kV}$.

\section{Apolipoprotein E Genotyping}

Genomic DNA was extracted from $50 \mathrm{mg}$ of cerebellar tissue and amplified by PCR using allele-specific oligonucleotide probes spanning positions 112 and 158 of the Apo E gene sequence. The PCR products were HhaI-restricted, and the fragments were separated by polyacrylamide electrophoresis. Genotypes were distinguished on the basis of differential HhaI band fragment mobilities as described by Hixson and Vernier (19).

\section{Quantification of Cortical A $\beta$ by Immunoassay}

Cerebral cortex fractions containing $A \beta$ were purified by fast performance liquid chromatography, and $A \beta$ peptide was quantified by immunoassay as described previously (20).

\section{Preparation of Leptomeninges}

Leptomeningeal tissue was prepared by partially transecting the larger vessels to facilitate the release of entrapped blood. The specimens then were rinsed 20 times with cold distilled water containing $0.01 \%$ sodium azide to promote hemolysis and prevent contamination. Blood vessels larger than 1-mm diameter were removed. The cleaned, colorless membranes of leptomeninges and the vascular network were then spread on disposable 15-cm Petri dishes and dried in an oven at $50{ }^{\circ} \mathrm{C}$ for $18 \mathrm{~h}$. The leptomeninges were stained with $1 \%$ aqueous Thioflavin-S, as described above.

\section{Preparation of Cerebral Cortex Vascular Tufts}

From a slice of cerebrum (parietal lobe), approximately 8- to 10-mm thick, the GM was dissected from the underlying WM and sectioned into small cubes measuring about 4 to $5 \mathrm{~mm}$. A total of 25 pieces of cerebral cortex from each individual case were stirred for approximately $24 \mathrm{~h}$ in 1 liter of lysis buffer ( $5 \%$ sodium dodecyl sulfate, $50 \mathrm{mM}$ Tris $\mathrm{HCl}, 3 \mathrm{mM}$ ethylenediaminetetraacetic acid [EDTA], pH 7.4). After $24 \mathrm{~h}$, pieces of the tissue were allowed to settle and the lysis buffer decanted. A 2nd liter of lysis buffer was added, and after complete brain tissue lysis, the white tufts of blood vessels were isolated by filtration through a nylon mesh $(0.12 \mathrm{~mm})$ mounted on a $10-\mathrm{cm}$ diameter plastic embroidery hoop. Those vessels retained on the nylon mesh were immediately released by vigorous shaking of the nylon mesh in 1 liter of distilled water containing $0.01 \%$ sodium azide. The vessel preparations were filtered twice more using the same technique. After 
Table 1. Study Subjects

\begin{tabular}{|c|c|c|c|c|c|c|}
\hline Case ID & Age/Sex & ApoE & White matter score & Plaque density & Break stage & NIA-Reagan Score \\
\hline \multicolumn{7}{|c|}{ Control cases } \\
\hline 1 & $94 / F$ & $3 / 3$ & 1 & Mod & III & ND \\
\hline 2 & $94 / M$ & $3 / 3$ & 1 & Mod & III & ND \\
\hline 3 & $85 / M$ & $3 / 3$ & 1 & None & $\|$ & ND \\
\hline 4 & $86 / F$ & $3 / 3$ & 0 & None & III & ND \\
\hline 5 & $89 / F$ & $3 / 3$ & 1 & Mod & IV & ND \\
\hline 6 & $76 / F$ & $3 / 3$ & 1 & Mod & I & ND \\
\hline 7 & $84 / M$ & $3 / 3$ & 0 & Mod & III & ND \\
\hline 8 & $78 / M$ & $3 / 3$ & 1 & None & I & ND \\
\hline 9 & $92 / \mathrm{M}$ & $3 / 3$ & 0 & Sparse & III & ND \\
\hline 10 & $74 / M$ & $3 / 3$ & 0 & Mod & IV & ND \\
\hline 11 & $86 / M$ & $3 / 3$ & 1 & None & $\|$ & ND \\
\hline 21 & $80 / F$ & $3 / 4$ & 1 & Mod & III & ND \\
\hline 22 & $83 / F$ & $3 / 4$ & 1 & Mod & $\|$ & ND \\
\hline 23 & $86 / F$ & $3 / 4$ & 1 & Mod & III & ND \\
\hline 24 & $85 / F$ & $3 / 4$ & 0 & None & III & ND \\
\hline 25 & $69 / \mathrm{M}$ & $3 / 4$ & 1 & Sparse & 1 & ND \\
\hline 26 & $73 / M$ & $3 / 4$ & 0 & None & III & ND \\
\hline \multicolumn{7}{|c|}{ Alzheimer's disease cases } \\
\hline 51 & $93 / F$ & $3 / 3$ & 0 & Freq & VI & High \\
\hline 52 & $60 / F$ & $3 / 3$ & 1 & Freq & VI & High \\
\hline 53 & $90 / F$ & $3 / 3$ & 2 & Freq & $\vee$ & High \\
\hline 54 & $96 / F$ & $3 / 3$ & 3 & Mod & $\vee$ & High \\
\hline 55 & $80 / F$ & $3 / 3$ & 3 & Freq & $\mathrm{VI}$ & High \\
\hline 56 & $85 / F$ & $3 / 3$ & 2 & Freq & $\mathrm{V}$ & High \\
\hline 61 & $91 / \mathrm{M}$ & $3 / 4$ & 2 & Freq & $\mathrm{V}$ & High \\
\hline 62 & $87 / M$ & $3 / 4$ & 0 & Freq & V & High \\
\hline 63 & $60 / M$ & $3 / 4$ & 0 & Freq & VI & High \\
\hline 64 & $84 / M$ & $3 / 4$ & 1 & Freq & $\mathrm{V}$ & High \\
\hline 65 & $89 \mathrm{~F}$ & $3 / 4$ & 3 & Freq & $\mathrm{V}$ & High \\
\hline 66 & $87 / M$ & $3 / 4$ & 2 & Freq & $\mathrm{V}$ & High \\
\hline 71 & $79 / M$ & $4 / 4$ & 1 & Mod & $\mathrm{V}$ & High \\
\hline 72 & $81 / F$ & $4 / 4$ & 0 & Freq & $\mathrm{VI}$ & High \\
\hline 73 & $79 / F$ & $4 / 4$ & 3 & Freq & IV & $\ln t$ \\
\hline 74 & $68 / M$ & $4 / 4$ & 1 & Freq & VI & High \\
\hline 75 & $77 / M$ & $4 / 4$ & 0 & Freq & VI & High \\
\hline 76 & $87 / M$ & $4 / 4$ & 2 & Mod & IV & Int \\
\hline 77 & $79 / M$ & $4 / 4$ & 2 & Freq & $\mathrm{V}$ & High \\
\hline 78 & $80 / M$ & $4 / 4$ & 3 & Freq & VI & High \\
\hline 79 & $80 / F$ & $4 / 4$ & 1 & Freq & VI & High \\
\hline 80 & $93 / F$ & $4 / 4$ & 2 & Freq & VI & High \\
\hline 81 & $84 / M$ & $4 / 4$ & 1 & Freq & VI & High \\
\hline 82 & $83 / M$ & $4 / 4$ & 1 & Freq & $\mathrm{VI}$ & High \\
\hline
\end{tabular}

Mod, moderate; Freq, frequent; Int, intermediate; ND, nondemented.

settling at room temperature for $16 \mathrm{~h}$, the water phase was removed by aspiration and the vascular suspension, approximately $30 \mathrm{~mL}$ in volume, was poured onto a Petri dish. The vascular tufts were removed from the Petri dish and spread onto a glass microscope slide. The blood vessel preparations were air dried at $60{ }^{\circ} \mathrm{C}$ for $6 \mathrm{~h}$, fixed in absolute ethanol for $30 \mathrm{~min}$, rinsed with distilled water, and Thioflavin-S stained. The stained prepa- rations were observed using an epifluorescence microscope (Zeiss Axiophot) equipped with a 450 to $490 \mathrm{~nm}$ excitation filter and a $520 \mathrm{~nm}$ barrier filter.

\section{Morphometric Assessment of État Criblé-like Lesions}

Brain tissue sections stained with $\mathrm{H} \& \mathrm{E}$ were viewed at $25 x$ magnification with the Leica DMLB microscope system (Leica 

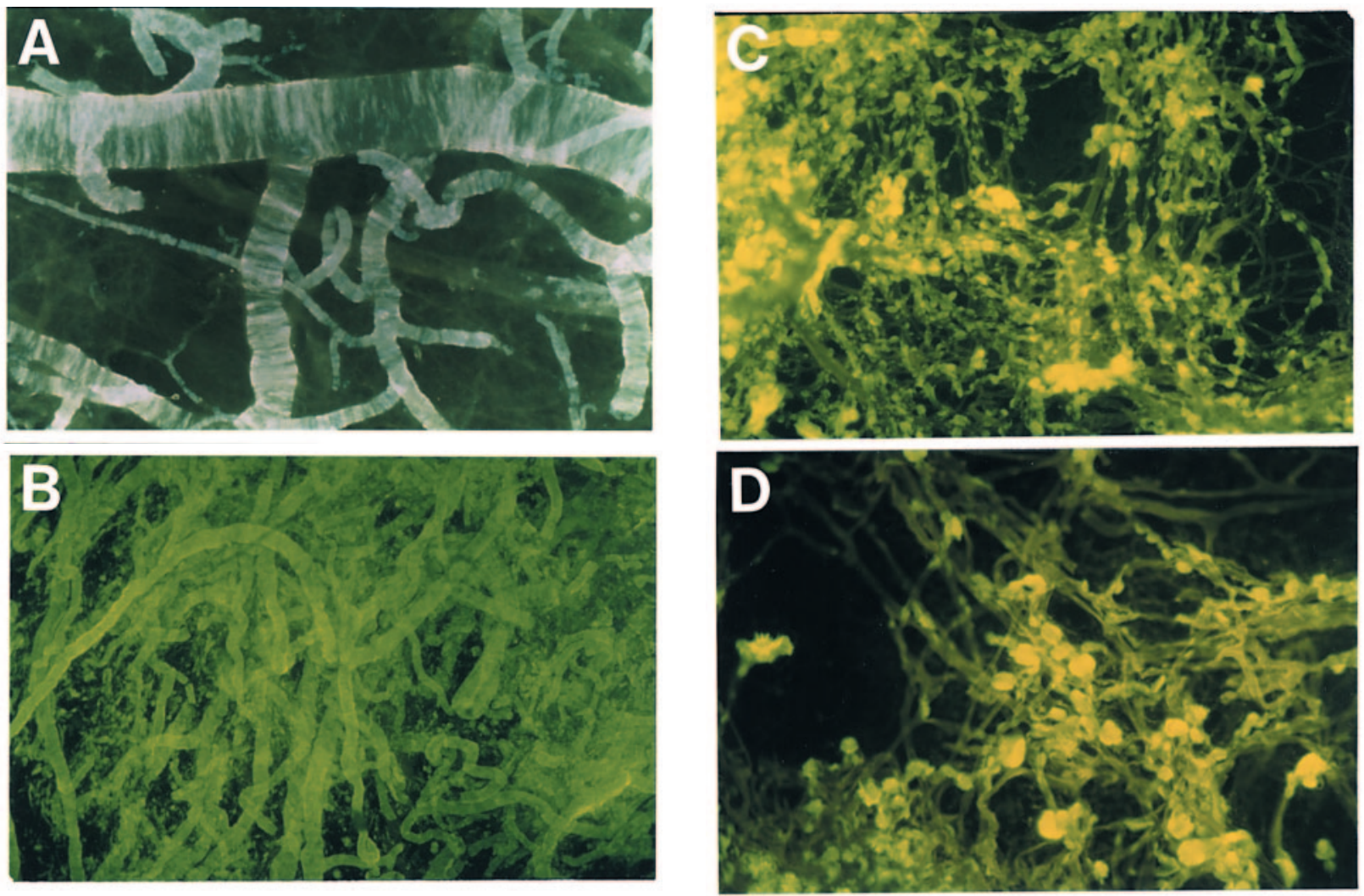

Figure 1. Thioflavin-S-stained whole-mounted tufts of cortical vessels after complete brain parenchyma lysis by EDTA-SDS. A: Ring-like amyloid deposits in leptomeningeal arterial walls. The pattern of deposition follows the orientation of the smooth muscle cells. Magnification $25 x$. B: Tufts of corticalpenetrating arteries heavily loaded with amyloid from an AD patient with Apo E $\varepsilon 4 / \varepsilon 4$ genotype. The perivascular spaces are probably occluded in the saturated amyloid vessels, and in some cases may be totally destroyed by the encroaching amyloid, thus hindering the elimination of interstitial fluid. Magnification 100x. C: Cortical arterioles and capillaries showing an abundant deposition of amyloid cores, in an individual with Apo $\mathrm{E} \varepsilon 4 / \varepsilon 4$ genotype, at different stages of development that are intimately linked to the basal laminae of the vessels. The profuse deposits of amyloid at the arteriolar/capillary junction may block the openings of the perivascular spaces that drain the brain's interstitial fluid. Magnification 200x. D: At a higher magnification, each of the fluorescent blebs represents a fully developed globular deposit of amyloid evenly spread around and constricting the microvessel, probably creating areas of ischemia and alterations in blood-brain barrier. Magnification 400x.

Microsystems AB, Ernst-Leitz-Strasse, Wezlar, Germany) with a $100 \mathrm{~W}$ transmitted light source. The whole area of each slide was scanned for evidence of dilated perivascular spaces in the WM; their outlines were digitized with an Optronics Magnafire SP camera (model s99805) using the Optronics Magnafire SP software program (Optronics, Goleta, CA) and stored in TIF format. All measurements were carried out using Image-Pro Express software v. 4.0 (Media Cybernetics, Silver Springs, MD), which was calibrated to $25 \times$ magnification. The diameter of each enlarged perivascular space, that is, larger than $1 \mathrm{~mm}$ in perimeter, and the blood vessel within were measured using the straight line tool and computer mouse. The data were transferred to Microsoft Excel $^{\circledR}$, and the mean ratios of perivascular space diameters to blood vessel diameters for each case were calculated and then grouped by ApoE genotype and diagnosis.

\section{RESULTS}

\section{Cerebral Amyloid Angiopathy}

A detailed analysis of entire tufts of cerebral vessels spanning the GM of the cerebral cortex was performed on preparations of cor- tex after complete cell lysis by the combined action of EDTA-SDS. No vascular amyloid was detected by Thioflavin-S-staining in control brains, but amyloid deposits were present in AD brains. All vessels less than $500 \mu \mathrm{m}$ in diameter had amyloid deposits (Figure 1A and 1B). The deposits of amyloid tend to disappear in larger leptomeningeal arteries. The arteriolar and capillary vessels demonstrated a unique abundance of discreet 'core-like' amyloid deposits firmly attached to the vessel wall and, in many cases, the cores appeared to be fused together (see Figure 1C and 1D). All the spherical amyloid cores associated with capillaries displayed a brush-like surface. On average, there was a moderate amount of amyloid associated with the vessels of the cerebral cortex in $\mathrm{AD}$ patients with $\mathrm{ApoE} \varepsilon 3 / \varepsilon 3$ and $\varepsilon 3 / \varepsilon 4$ genotypes. However, the most severely affected individuals, in terms of amyloid load, were those with ApoE $\varepsilon 4 / \varepsilon 4$ genotype (see Figure 1). Whole mount preparations of all $\varepsilon 4 / \varepsilon 4 \mathrm{AD}$ subjects revealed an overwhelming amount of vascular amyloid in the cerebral cortex. Electron microscopy of the GM arteries showed a virtual disappearance of smooth muscle cells from artery walls containing large amounts of amyloid and obliteration of the perivascular spaces (Figures 1A and 2B, 2C, and 2D). 


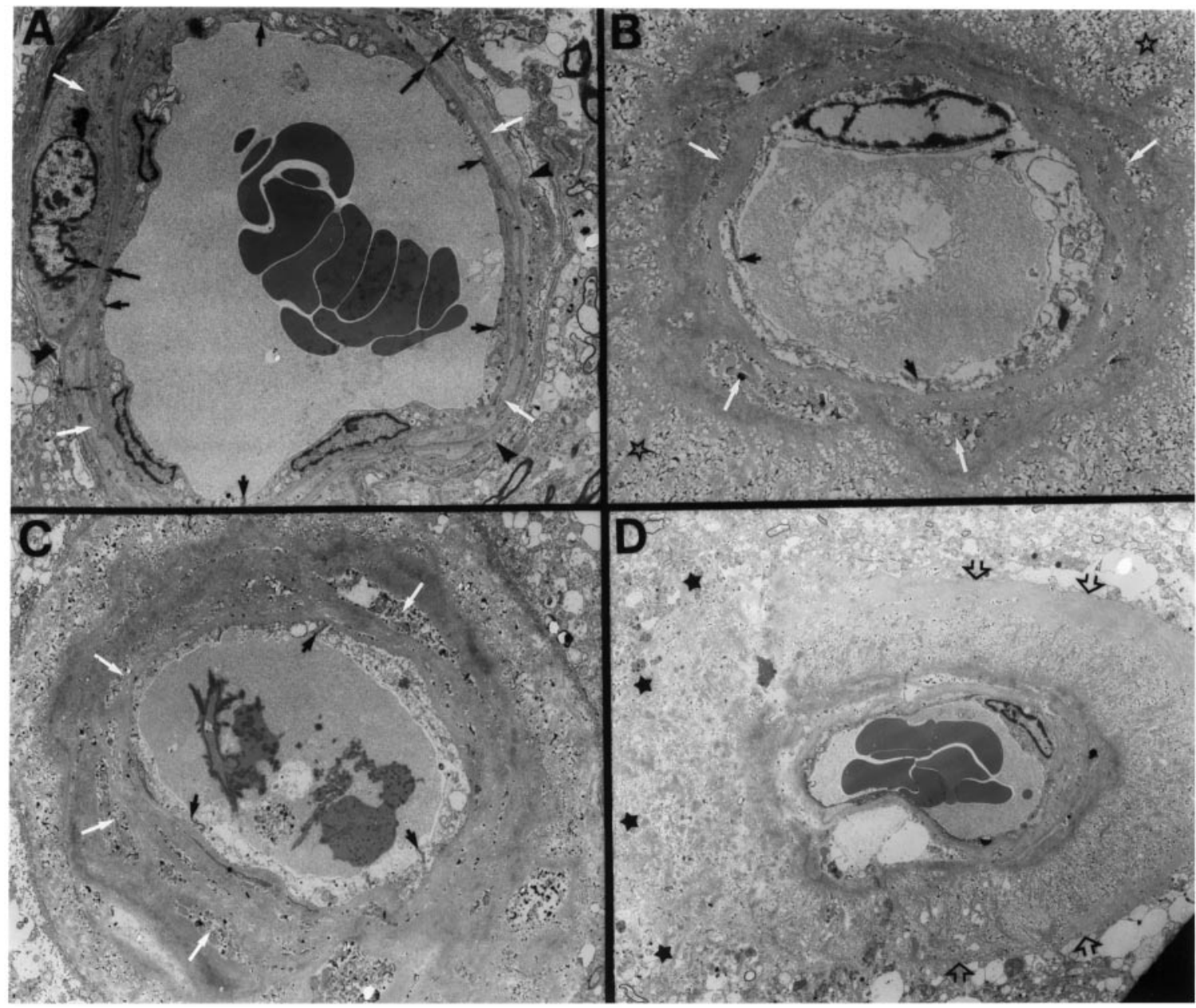

Figure 2. Electron micrographs of a normal cortical artery and of arteries destroyed by amyloid deposition in 3 AD cases homozygous for Apo $E$ \&4/ع4. A: An electron micrograph of a small normal cortical artery. The endothelial cells are joined by tight junctions (small arrows) that participate in the blood-brain barrier. Both their nuclei and thin cytoplasm have a normal morphology. These cells are in contact with the subjacent basal lamina (opposing large arrows). The smooth muscle cells (white arrows) have a normal-looking fibrillar cytoplasm, and a typical nucleus is seen on the left margin of the photograph. The thin periarterial space (black arrowheads) contains the extracellular matter of the adventitia and is on its outer margin limited by a single layer of pial cells (8). Magnification 5500x. B and C: Small cortical arteries surrounded by heavy amyloid deposition. The endothelial cells are apparently swollen. The tight junctions have an abnormal morphology (black arrows). The dense amyloid deposits are fused with the basal lamina of the vascular cells. The myocytes have vanished or are reduced to a collection of cellular debris (white arrows) that is enclosed by concentric layers and peripheral wisps of amyloid surrounded by a large amount of tissue debris (open stars). The tunica adventitia, perivascular spaces, and the glia limitans have degenerated as the result of heavy amyloid deposition. Magnification 4300x and magnification 3500x, respectively. D: An abnormal cortical artery with abundant fine wisps of amyloid perpendicular to the main axis of the vessel. The amyloid bundles, interspersed by large amounts of cellular debris, pushed out the glia limitans (open arrows). On the left margin of the micrograph, the amyloid has destroyed the glia limitans invading the surrounding cortical tissue (black stars). All myocytes and the tunica adventitia have been replaced by amyloid fibrils and debris. Magnification 2000x. One may assume from the extensive vascular pathology that the delivery of oxygen and nutrients as well as arterial contractility are largely compromised.

In $\mathrm{AD}$ cases, most of the arteries in the leptomeninges, up to a diameter of $500 \mu \mathrm{m}$, demonstrated a heavy load of ring-like deposits similar to those seen in the cerebral cortical arteries (see Figure 1A). However, in those individuals with ApoE $\varepsilon 3 / \varepsilon 3$ and $\varepsilon 3 / \varepsilon 4$ genotypes, the distribution of amyloid in the leptomeningeal vessels was segmental; that is, portions of artery with a heavy load of amyloid were interspersed with lengths of vessel that were free of amyloid. As in the cerebral cortex, those individuals with ApoE $\varepsilon 4 / \varepsilon 4$ genotypes had a more prominent amount of vascular amyloid involving almost all the small $(<500 \mu \mathrm{m})$ leptomeningeal arteries. As the leptomeningeal arteries increased in diameter, the amyloid load gradually declined to isolated streaks associated with the more peripheral layers of the tunica media. The large branches of the circle of Willis were entirely devoid of microscopically visible (Thioflavin-S positive) amyloid. Occasionally in the leptomeninges, areas of old microhemorrhages were revealed by the presence of hemosiderin deposits along vessels burdened with amyloid (data not shown).

\section{Dilation of Perivascular Spaces in AD}

Macroscopic inspection of brain sections from ND controls (Figure $3 \mathrm{~A}$ ) and from individuals with $\mathrm{AD}$ (see Figure $3 \mathrm{~B}$ ) revealed a 


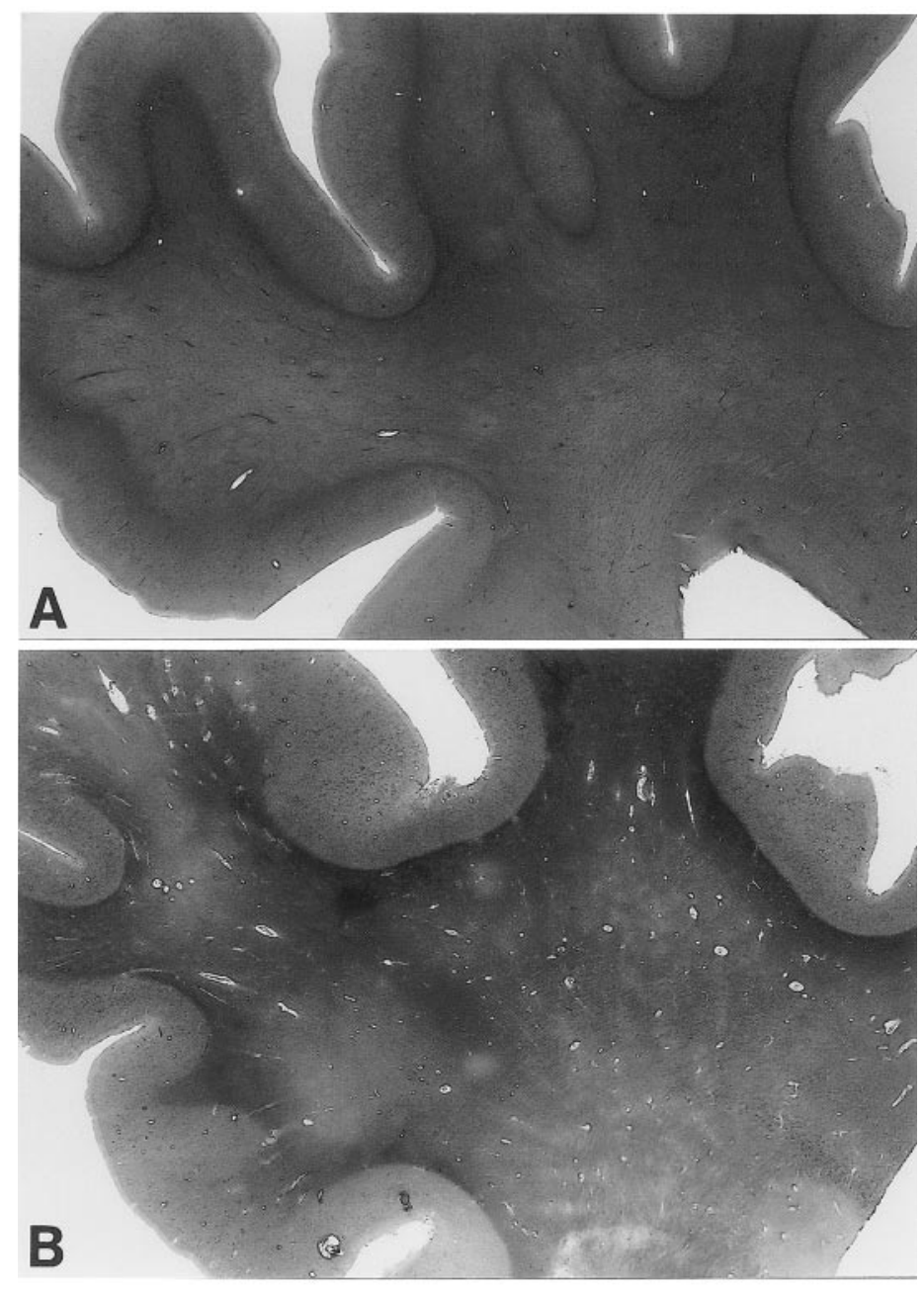

Figure 3. Hematoxylin- and eosin-stained superior frontal gyrus and underlying WM sections from an ND individual and an AD case. Magnification: about 2.5x. A: Cerebral section from a 74-y-old ND individual with ApoE $\varepsilon 3 / \varepsilon 3$ genotype, who died as the result of metastatic prostate cancer. The WM appears homogeneously stained without noticeable dilated periarterial spaces. B: Shows a section from an $80-y$-old AD patient with ApoE $\varepsilon 4 / \varepsilon 4$ genotype. The numerous arteries with enlarged perivascular spaces are evident throughout the entire extent of the WM, whereas the GM is comparatively free of these alterations. The paler blotches in the WM represent areas of myelin rarefaction.

large number of dilated perivascular spaces in the WM of AD cases. The dilated perivascular spaces were more prominently displayed by light microscopy (Figure 4). Very few dilated perivascular spaces were seen in control material. Areas of rarefaction in the WM were more abundant in AD than in the control cases. As shown in Table 1, the average WM rarefaction score was significantly higher in the AD cases (mean $=1.5$, range 0 to 3 ) than in the control cases (mean $=0.65$, range 0 to $1 ; P=0.004)$.

\section{Quantitation of $\mathbf{A} \beta$ and Dilated Perivascular Spaces in the White Matter}

Quantitation of total cortical $A \beta$ (A $\beta 40$ and $A \beta 42$ ) by immunoassay from ApoE $\varepsilon 4 / \varepsilon 4$ AD subjects yielded an average of $54 \mu \mathrm{g} / \mathrm{g}$ of tissue with 2 of 3 of the $A \beta$ peptides ending at residue 42 ; this is supported by our previous observations (21). Total $\mathrm{A} \beta$ in the cortex quantified by immunoassay in the ApoE $\varepsilon 3 / \varepsilon 4$ and $\varepsilon 3 / \varepsilon 3$ cohorts showed mean values of 26 and $35 \mu \mathrm{g} / \mathrm{g}$ of tissue, respectively, with a predominance of $\mathrm{A} \beta$ 42. In these cases, core-like and ring-like amyloid deposition prevailed, resembling the type 2 deposits described by Thal and others (22). In contrast to the $\mathrm{AD}$ subjects, the total $\mathrm{A} \beta$ in control individuals carrying the ApoE $\varepsilon 3 / \varepsilon 3$ and $\varepsilon 3 / \varepsilon 4$ genotypes only amounted to 3 and $4 \mu \mathrm{g} / \mathrm{g}$ of tissue, respectively.

Total $\mathrm{A} \beta$ in GM of AD patients was 12 times greater than in control cases (Figure 5A). The amount of $\mathrm{A} \beta$ was significantly higher in the AD patients with ApoE $\varepsilon 4 / \varepsilon 4$ than in those AD individuals with ApoE $\varepsilon 3 / \varepsilon 4$ and $\varepsilon 3 / \varepsilon 3$ genotypes (see Figure $5 \mathrm{~A}$ ). Likewise, the number of perivascular spaces in WM as well as the ratio of the diameters of perivascular spaces over the diameters of centrally located blood vessels were significantly higher in the AD cohort when compared with ND controls (see Figure 5B and 5C). When the AD cohorts were grouped by ApoE genotype, there was a positive correlation between the average number of enlarged perivascular spaces and the average cortical concentration of $A \beta$ (see Figure 5D). The ratio of the perivascular space diameter over blood vessel diameter also had a significant positive correlation with the cortical $\mathrm{A} \beta$ concentration (see Figure $5 \mathrm{E}$ ) and the number of enlarged perivascular spaces (see Figure 5F). In contrast to the ND population, the AD cohort had an increased load of cortical A $\beta$, a higher count of distended perivascular spaces (état criblé-like lesions). In addition, the distended perivascular spaces were larger relative to the size of their respective blood vessels. Furthermore, individuals with the ApoE $\varepsilon 4 / \varepsilon 4$ genotype have more of the aforementioned pathology than those with 1 or no ApoE $\varepsilon 4$ alleles.

\section{Relationship Between Dilated Perivascular Spaces and Cerebral Atrophy}

We also explored the possibility that enlargement of perivascular spaces may result from retraction of WM due to overall brain atrophy. Because there is a significant difference in brain size between genders, these cohorts were evaluated separately. No correlation was found between brain weight and the average number of enlarged perivascular spaces in the WM or between brain weight and the average ratio of perivascular space diameter to blood vessel diameter.

\section{DISCUSSION}

Our results suggest that there is a correlation between the severity of cortical and leptomeningeal CAA, the amount of $A \beta$ in the cerebral cortex, and the accumulation of ISF in dilated perivascular spaces (état criblé-like lesions) in AD WM. Conventional histological techniques do not allow full evaluation of CAA. By stripping the leptomeningeal arteries from the cortex and then removing all traces of blood and Thioflavin-S staining, a clear picture of the extent of amyloid involvement of cerebral vessels emerges. Leptomeningeal arteries with CAA have a ring-like pattern of $A \beta$ deposition that follows the orientation of smooth muscle cells within the media. A similar pattern is seen in the larger cortical arteries. A ring-like distribution of $A \beta$ in which whole 


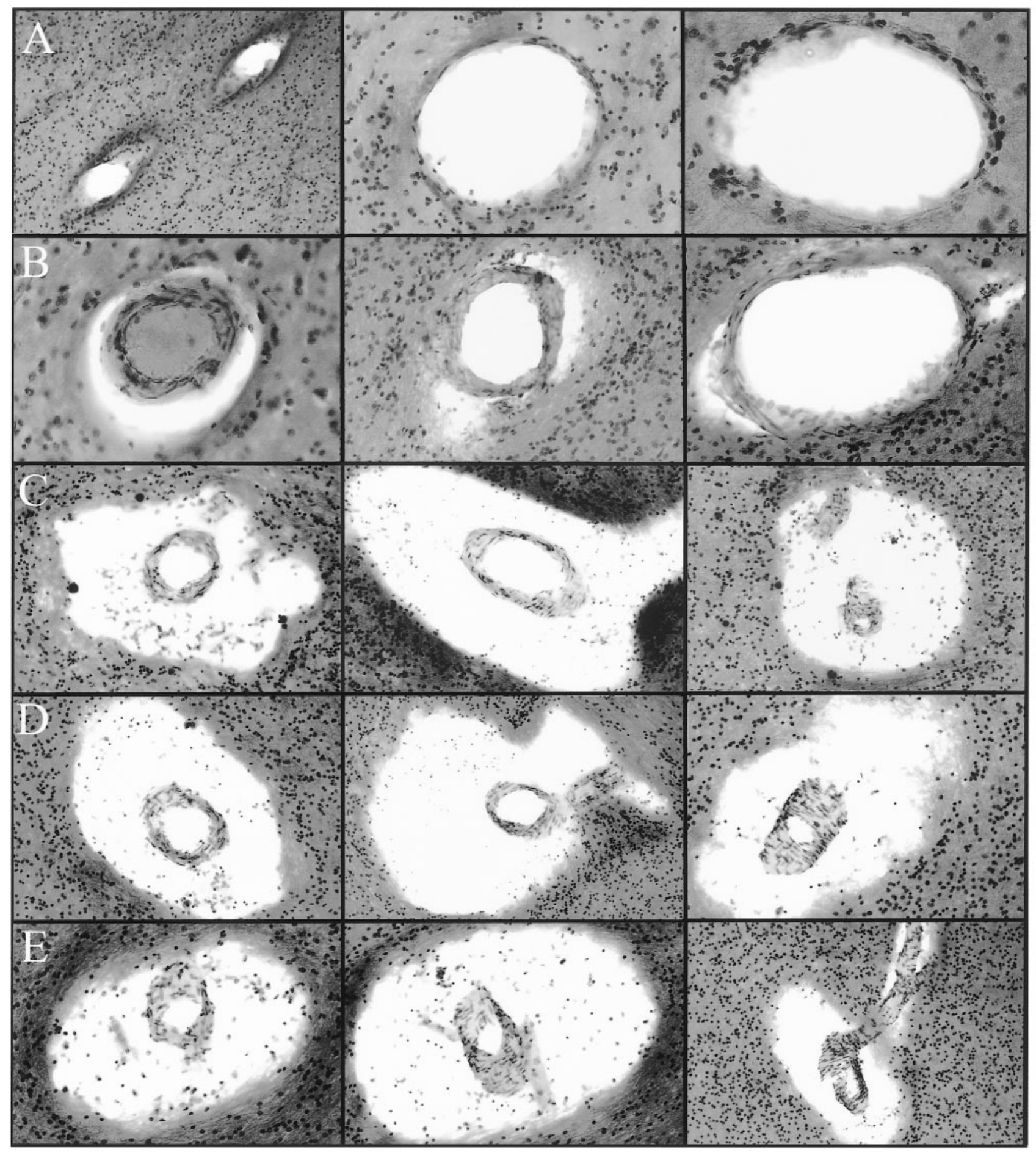

Figure 4. Perivascular spaces in Alzheimer's disease white matter. Row A: The fine periarterial spaces are not normally visible at light microscopic level. Magnifications: left and center 100x; right 200x. Row B: The periarterial spaces appear to be slightly dilated. Magnifications: left 200x; center and right 100x. Rows C, D, and E demonstrate a series of severely distended perivascular spaces. The centrally located arteries apparently have a normal morphology. All captions were taken at 100x. All histological slides were stained with hematoxylin and eosin.

lengths of vessel are virtually replaced by $\mathrm{A} \beta$ is seen in smaller arteries and arterioles. Core-like deposits of $A \beta$ form in capillary walls, and they are often arranged in confluent rows. It is difficult to assess the effect of arterial and capillary CAA on the blood supply of the cerebral cortex. We observed that some of the capillar- ies are occluded by amyloid deposits. In other studies, capillary lumina remained patent even in the presence of heavy deposition of $A \beta$ in the capillary basement membrane (17). Even if occlusion did occur, extensive capillary anastomoses exist within the cortex (16), making any physiological effects difficult to predict. 

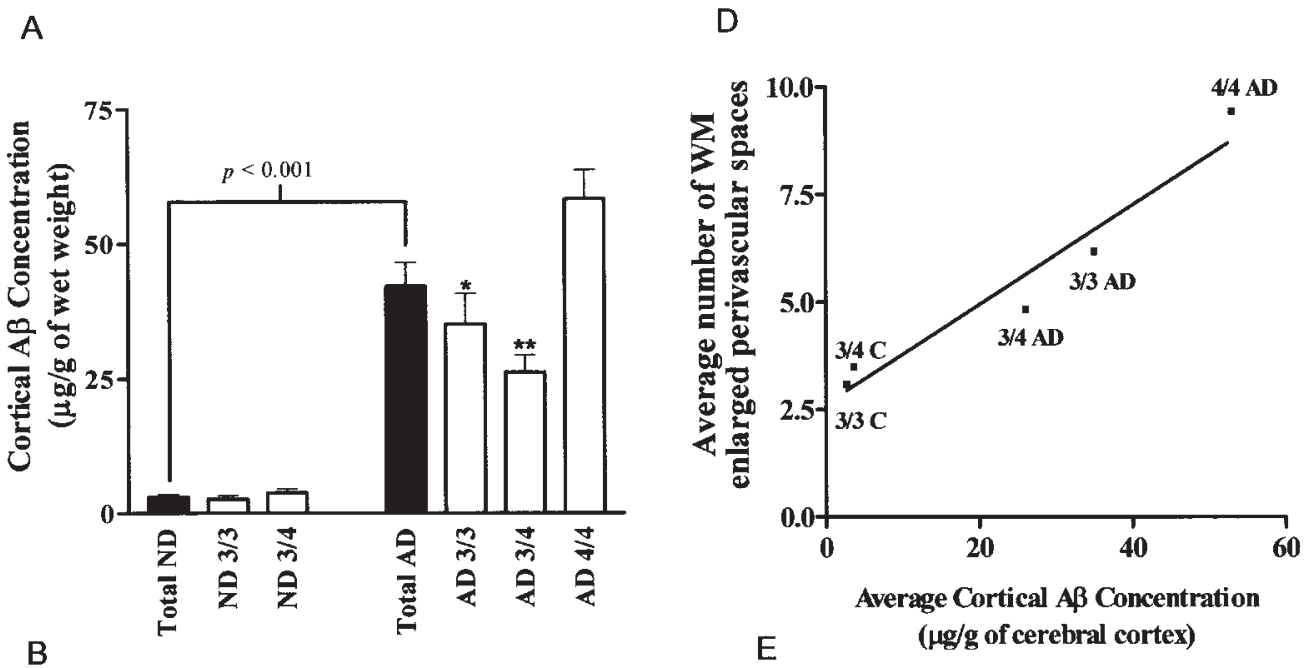

B
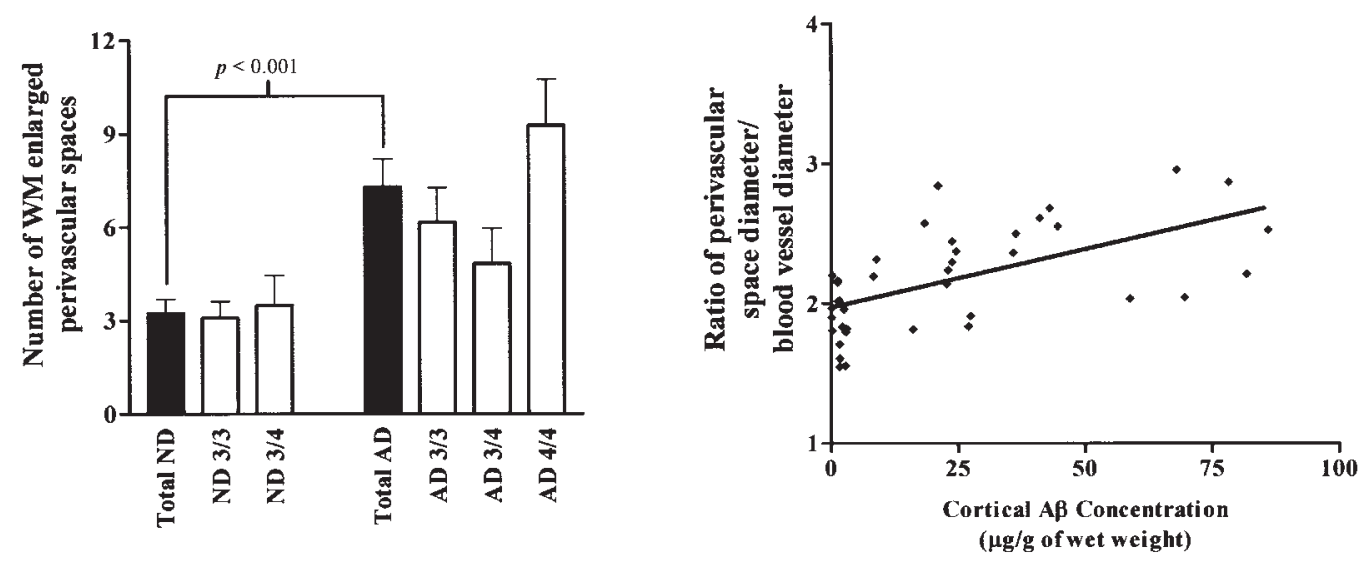

C

$\mathrm{F}$
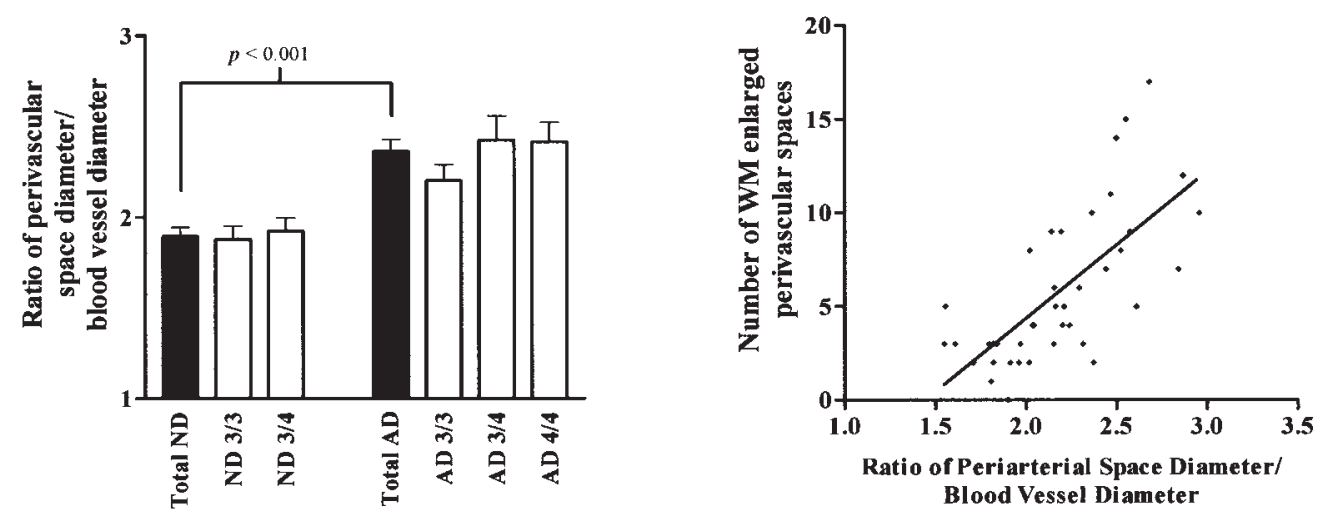

Figure 5. Quantitative and statistical analyses among the 3 variables investigated in this study: cortical A $\beta$ concentrations, number of dilated perivascular spaces (état criblé-like lesions), and ratio of perivascular space diameter/blood vessel diameter. In the 3 left panels, $\mathbf{a}$ and $\square$ histograms represent the total numbers of ND and AD individuals and these populations divided by Apo E genotype, respectively. The 3 panels on the right side of the figure demonstrate the linear correlations and $P$ values among the 3 studied variables. A: The total levels of cortical A $\beta$ in ND and AD subjects. $P$ value represents the 2 -tailed unpaired Student $t$-test probability. ${ }^{*}$ and ${ }^{* *}$ represent $P<0.05$ and $P<0.01$, respectively, in post-hoc analysis (NewmanKeuls Multiple Comparison Test) after 1-way analysis of variance demonstrated that there was a significant difference among the AD subgroups. B: The average number of WM enlarged perivascular spaces (état criblé-like lesions) in the ND and AD groups. $P$ value represents the 2-tailed unpaired Student t-test probability. C: Mean ratios of perivascular space diameter/blood vessel diameter observed in ND and AD cohorts. $P$ value represents the 2-tailed unpaired Student $t$-test probability. $D$ : Linear correlation $(R=0.97)$ between the mean number of WM enlarged perivascular spaces and average levels of cortical A $\beta$ when the AD and control subjects were grouped by Apo E genotype. E: Linear correlation between the ratio of perivascular space diameter to blood vessel diameter and the total levels of cortical $A \beta(R=0.55 ; P<0.001)$. F: Linear correlation between the mean number of WM enlarged perivascular spaces and the ratio of perivascular space diameter to blood vessel diameter $(R=0.70 ; P<0.001)$. 
A

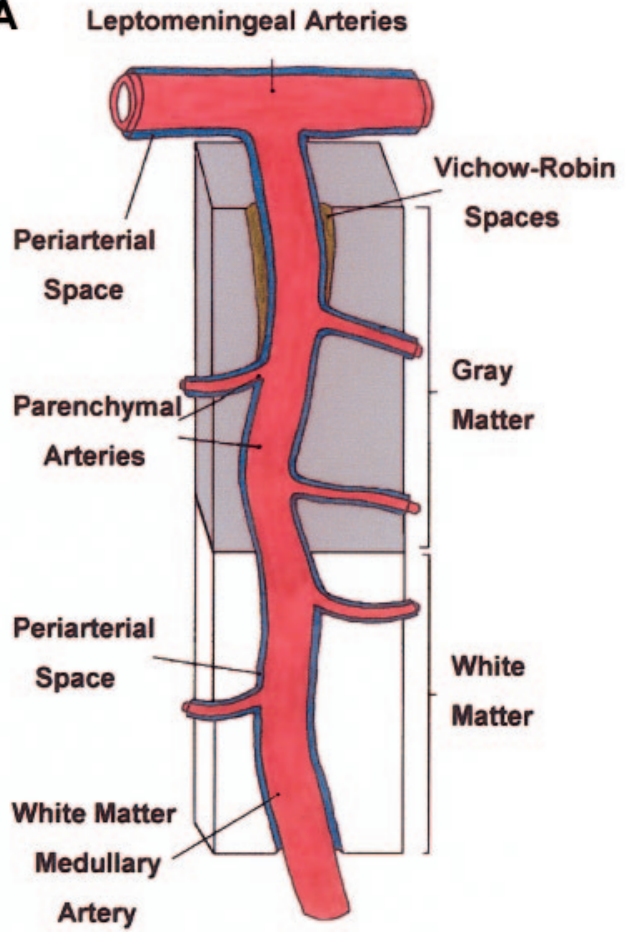

B

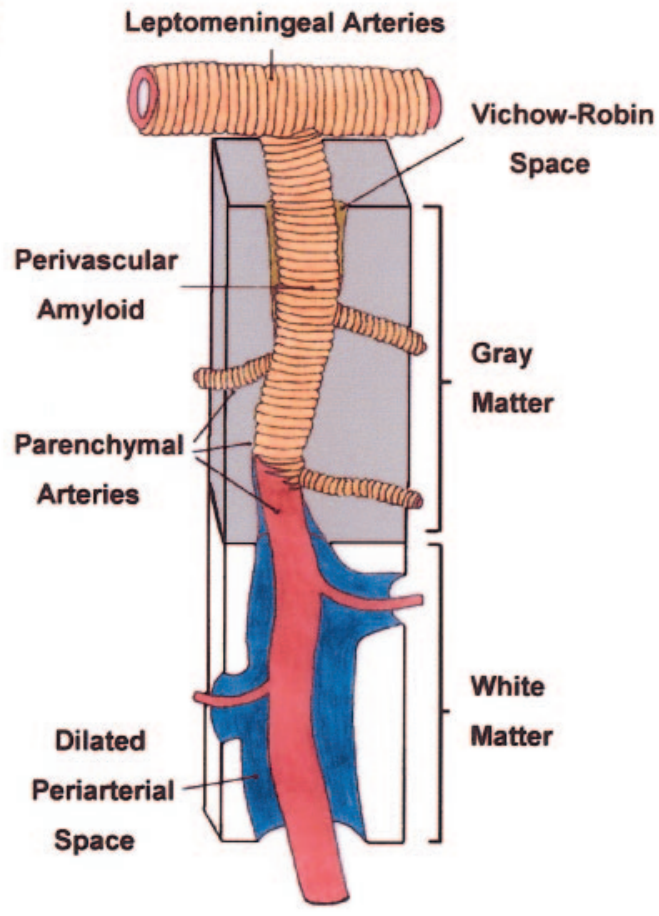

Figure 6. Diagram suggesting the morphological changes that lead to enlargement of WM perivascular spaces. A: Under normal conditions, the brain's interstitial fluid is collected by the patent periarterial spaces around the blood vessels of the WM, GM, and leptomeninges. B: The leptomeningeal and perforating cortical arteries supplying the brain GM and WM are surrounded by heavy amyloid deposits that destroy the vascular walls and block the perivascular spaces at the level of the cerebral cortex and leptomeninges. This obstruction results in WM congestion and stasis of interstitial fluid with simultaneous dilation of the WM perivascular spaces. The greater density and rigidity and lesser compliance of the GM matter leave this tissue substantially less affected than the WM.

Accumulation of $A \beta$ in the cerebral cortex and cerebral vessels correlates with dilation of perivascular spaces in the underlying WM. Thus the number of dilated perivascular spaces in Alzheimer brains is significantly greater than in ND individuals, and the number of dilated spaces is proportional to the level of $A \beta$ in the overlying cortex and associated arteries. Furthermore, $\mathrm{A} \beta$ concentrations in the cortex were higher for ApoE $\varepsilon 4 / \varepsilon 4$ cases than in other ApoE genotypes. Total A $\beta$ load in the WM was much higher in Alzheimer's disease than in ND individuals, (20) which suggests that $\mathrm{A} \beta$ elimination from the WM is reduced in AD.

In the present and previous investigations, we have observed that the amount of $A \beta$ deposited along the cortical and leptomeningeal vessels directly correlates with Apo E genotype (12). In AD, the relative amount of $A \beta$ in these vessels decreases as follows: $\varepsilon 4 / \varepsilon 4>>\varepsilon 3 / \varepsilon 3>\varepsilon 3 / \varepsilon 4$. It appears that slowly evolving occlusion of the perivascular spaces in the cortical and leptomeningeal arteries results in dilation of perivascular spaces in the WM, that is, état criblé, and subclinical chronic edema of the WM parenchyma. The extent of the edema is related to tissue compliance, which is greater in the WM than in the GM and greater in the deep WM than in the superficial WM (23). In humans, the WM of the centrum semiovale is supplied by long penetrating medullary arteries that are a continuation of cortical perforating vessels derived from the leptomeningeal arteries (16) and by perforating arteries directly derived from the circle of Willis (24).
Little attention has been paid in the past to the effects of CAA on WM in AD despite the evidence from Duvernoy and others (16) that branches of leptomeningeal arteries supply the cerebral WM as well as the cortex. Although CAA is rarely seen in arteries in the WM, impedance of ISF flow from the WM still could occur due to involvement of leptomeningeal and cortical arteries by CAA. It is of interest for the future development of immunotherapy for Alzheimer's disease that some of the patients treated by $A \beta$ immunization develop severe rarefaction and significant increase of ISF in the WM. In 1 post-mortem case, the WM edema was accompanied by severe CAA (25).

État criblé has been classically described as the consequence of dilation of perivascular spaces due to enlargement, distortion, and formation of loops in cerebral arterioles and small arteries that result from aging, hypertension, or a combination of both factors. In our AD cohort, we rarely observed the characteristic thickening and hyalinosis of artery walls commonly seen in the WM as the état criblé associated with hypertension (14). Rather, in the état criblé-like condition of $\mathrm{AD}$, most of the $\mathrm{WM}$ arteries appear to have a normal configuration.

The état crible quantified in the present study resembles the dilated perivascular spaces occasionally seen in the WM of younger individuals, with or without an accompanying dementia (26). Dilated perivascular spaces also are seen in the GM of the basal ganglia as état lacunaire but rarely if at all in the cortex. Giant 
lacunes in the basal ganglia or in the mid-brain may obstruct CSF flow and result in hydrocephalus (27). It has been suggested that such lacunes are due to blockage of ISF drainage pathways (28). The anatomy of perivascular spaces of arteries in the WM appears to be similar to that of arteries in the basal ganglia but differs from cortical vessel $(26,28)$. Thus the structure of the perivascular spaces may play a key role in the preferential formation of état lacunaire in the basal ganglia and état criblé in the WM.

We have found that the WM of AD subjects is in general more fragile and softer than the WM of control elderly individuals, which under pressure has a higher resilience (20). From a biochemical viewpoint, the AD WM has significantly less cholesterol and myelin proteins and an elevated amount of $\mathrm{A} \beta$ peptides, even though parenchymal amyloid deposits and obvious perivascular amyloid accumulations are lacking (20). Ultrastructural studies have demonstrated that the AD WM, relative to controls, has a decreased number of axons and myelin levels, which are accompanied by the presence of large astrocytes with swollen-like cytoplasm (B Bohrmann and AE Roher, unpublished results). The astrocytes, by virtue of being interconnected by gap junctions, apparently regulate the amount of water and ions present in the extracellular space of relatively large areas of the brain (29). We postulate that obstruction of ISF drainage caused by deposition of amyloid in cortical and leptomeningeal blood vessel walls not only dilates the perivascular spaces in the WM and produces ISF stasis but also forces the sub-ependymal astrocytes to eliminate excessive amounts of water into the ventricles. The production of CSF by the choroid plexi in a normal adult is around $600 \mathrm{~mL} / \mathrm{d}$, whereas in $\mathrm{AD}$ patients it is substantially reduced to about $300 \mathrm{~mL} / \mathrm{d}(30)$. In view of these observations, it is possible that the remarkable enlargement of the lateral ventricles in $\mathrm{AD}$ results from, among other factors, the sustained drainage of extra fluid from WM into the lateral ventricles. Thus, the ventricles may act as a sink for interstitial WM fluid that could not be cleared through the amyloid-obstructed perivascular spaces.

In conclusion, the results of this study strongly support our hypothesis that compromised drainage of interstitial fluid from the $\mathrm{WM}$ in $\mathrm{AD}$ is due to blockage of the cortical and leptomeningeal perivascular spaces by amyloid deposition, thereby causing dilatation of perivascular spaces in the WM (Figure 6). Examination of $\mathrm{AD}$ vessel topology has clarified the pattern of vascular amyloid deposition and suggests a possible pathophysiological chain of events. Our analyses suggest that dysfunctional A $\beta$ transport and attendant vascular amyloid deposition results in an impairment of brain fluid dynamics and leads to some of the deleterious changes linked to advanced AD. Fundamental structural changes in the integrity of the arterial tree may be the key factor initiating amyloid deposition (31). As the stability of the microvasculature and the blood-brain barrier declines with normal aging or through ischemic and traumatic injury, amyloid nucleation sites become available in the vasculature. Continued and relentless accretion of $A \beta$ along the cortical and leptomeningeal vessels may result in the ultimate impairment of the entire regional circulation, causing cerebral ischemia, stasis of interstitial fluid, and chronic subclinical edema of WM. These findings underscore the complex and multifactorial nature of AD pathology.

\section{ACKNOWLEDGMENTS}

This study was supported by National Institutes of Health grants AG 17490, AG 18345, and NF 38674 and by the State of Arizona Alzheimer's Research Center.

Address correspondence and reprint requests to Alex E. Roher, Sun Health Research Institute, 10515 West Santa Fe Drive, Sun City, AZ 85351. Phone: 623-876-5465; fax: 623-876-5698; e-mail: alex.roher@ sunhealth.org.

Submitted November 14, 2002; accepted for publication February 3, 2003.

\section{REFERENCES}

1. Mirra SS et al. (1991) The consortium to establish a registry for Alzheimer's disease (CERAD). Part II: standardization of the neuropathologic assessment of Alzheimer's disease. Neurology 41:479-86.

2. Braak H, Braak E. (1991) Demonstration of amyloid deposits and neurofibrillary changes in whole brain sections. Brain Pathol. 1:213-6.

3. The National Institute on Aging and Reagan Institute Working Group on diagnostic criteria for the neuropathological assessment of Alzheimer's disease. (1997) Neurobiol. Aging 18:S1-2.

4. Weller RO. (1998) Pathology of cerebrospinal fluid and interstitial fluid of the CNS: significance for Alzheimer disease, prion disorders and multiple sclerosis. J. Neuropathol. Exp. Neurol. 57:885-94.

5. Weller RO, Massey A, Newman TA, Hutchings M, Kuo YM, Roher AE. (1998) Cerebral amyloid angiopathy: amyloid- $\beta$ accumulates in putative interstitial fluid drainage pathways in Alzheimer's disease. Am. J. Pathol. 153:725-33.

6. Weller RO, Kida S, Zhang ET. (1992) Pathways of fluid drainage from the brainmorphological aspects and immunological significance in rat and man. Brain Pathol. 2:277-84.

7. Yamada S, DePasquale M, Patlak CS, Cserr HF. (1991) Albumin outflow into deep cervical lymph from different regions of rabbit brain. Am. J. Physiol. 261:H1197-204.

8. Zhang ET, Inman CB, Weller RO. (1990) Interrelationships of the pia matter and the perivascular (Virchow-Robin) spaces in the human cerebrum. J. Anat. 170: 111-23.

9. Weller RO, Massey A, Kuo YM, Roher AE. (2000) Cerebral amyloid angiopathy: accumulation of $A \beta$ in interstitial fluid drainage pathways in Alzheimer's disease. Ann. N.Y. Acad. Sci. 903:110-7.

10. Cserr HF, Harling-Berg CJ, Knopf PM. (1992) Drainage of brain extracellular fluid into blood and deep cervical lymph and its immunological significance. Brain Pathol. 2:269-76.

11. Van Dorpe J, Smeijers L, Dewachter I. (2000) Prominent cerebral amyloid angiopathy in transgenic mice overexpressing the London mutant of human APP in neurons. Am. J. Pathol. 157:1283-98.

12. Lue LF et al. (1999) Soluble amyloid- $\beta$ peptide concentration as a predictor of synaptic change in Alzheimer's disease. Am. J. Pathol. 155:853-62.

13. McLean CA et al. (1999) Soluble pool of $A \beta$ amyloid as a determinant of severity of neurodegeneration in Alzheimer's disease. Ann. Neurol. 46:860-6.

14. van Swieten JC et al. (1991) Periventricular lesions in the white matter on magnetic resonance imaging in the elderly: a morphometric correlation with arteriolosclerosis and dilated perivascular spaces. Brain 114:761-74.

15. Kalaria RN. (2001) Advances in molecular genetics and pathology of cerebrovascular disorders. Trends Neurosci. 24:392-400.

16. Duvernoy HM, Delon S, Vannson JL. (1981) Cortical blood vessels of the human brain. Brain Res. Bull. 7:519-79.

17. Preston SD, Steart PV, Wilkinson A, Nicoll JAR, Weller RO. (2003) Capillary and arterial cerebral amyloid angiopathy in Alzheimer's disease: defining the perivascular route for the elimination of amyloid- $\beta$ from the human brain. Neuropathol. Appl. Neurobiol. Forthcoming.

18. Cho HS, Hyman BT, Greenberg SM, Rebeck GW. (2001) Quantitation of ApoE domains in Alzheimer disease brain suggests a role for ApoE in A $\beta$ aggregation. J. Neuropathol. Exp. Neurol. 60:342-9.

19. Hixson JE, Vernier DT. (1990) Restriction isotyping of human apolipoprotein E by gene amplification and cleavage with Hhal. J. Lipid Res. 31:545-8.

20. Roher AE et al. (2002) Increased A $\beta$ peptides and reduced cholesterol and myelin proteins characterize white matter degeneration in Alzheimer's disease. Biochemistry 41:1 1080-90.

21. Roher AE et al. (1993) $\beta$-amyloid $1-42$ is a major component of cerebrovascular amyloid deposits: implications for the pathology of Alzheimer disease. Proc. Nat. Acad. Sci. U.S.A. 90:10836-40.

22. Thal DR, Ghebremedhin E, Rub U, Yamaguchi H, Tredici KD, Braak H. (2002) Two types of sporadic cerebral amyloid angiopathy. J. Neuropathol. Exp. Neurol. 61:282-93. 
23. O'Brien MD. (1995) Ischemic cerebral edema. In: Brain Ischemia. Caplan LR (ed.) Springer, New York, pp. 43-50.

24. Waddington MM. (1974) Atlas of cerebral angiography with anatomic correlation. Boston: Little, Brown and Co. p 72-5.

25. Nicoll JAR, Wilkinson D, Holmes C, Weller RO (2003) Neuropathology of human Alzheimer's disease following immunization with amyloid- $\beta$-peptide. Neuropathol. Appl. Neurobiol. Forthcoming.

26. Vital C, Julien J. (1997) Widespread dilation of perivascular spaces: a leukoencephalopathy causing dementia. Neurology 48:1310-3.

27. Kanamalla US, Calabro F, Jinkins JR. (2000) Cavernous dilation of mesencephalic Virchow-Robin spaces with obstructive hydrocephalus. Neuroradiology 42:881-4.
28. Pollack $\mathrm{H}$, Hutchings $M$, Weller RO, Zhang ET (1997) Perivascular spaces in the basal ganglia of the human brain: their relationship to lacunes. J. Anat. 191 337-46.

29. Nilsson M, Thorlin T, Blomstrand F, Hansson E. (2000) The star shaped cells: astrocytes are involved in the pathogenesis and progress of neurological diseases. Lakartidningen 97:3604-10.

30. Silverberg GD et al. (2001) The cerebrospinal fluid production rate is reduced in dementia of the Alzheimer's type. Neurology 57:1763-6.

31. Yow HY, Weller RO. (2002) A role for cerebrovascular disease in determining the pattern of $\beta$-amyloid deposition in Alzheimer's disease. Neuropathol. Appl. Neurobiol. 28:149. 